\title{
COMPACTNESS WITHIN THE SPACE OF COMPLETE, CONSTANT $Q$-CURVATURE METRICS ON THE SPHERE WITH ISOLATED SINGULARITIES
}

\author{
JOÃO HENRIQUE ANDRADE, JOÃO MARCOS DO Ó, AND JESSE RATZKIN
}

\begin{abstract}
In this paper we consider the moduli space of complete, conformally flat metrics on a sphere with $k$ punctures having constant positive $Q$-curvature and positive scalar curvature. Previous work has shown that such metrics admit an asymptotic expansion near each puncture, allowing one to define an asymptotic necksize of each singular point. We prove that any set in the moduli space such that the distances between distinct punctures and the asymptotic necksizes all remain bounded away from zero is sequentially compact, mirroring a theorem of D. Pollack about singular Yamabe metrics. Along the way we define a radial Pohozaev invariant at each puncture and refine some a priori bounds of the conformal factor, which may be of independent interest.
\end{abstract}

\section{INTRODUCTION}

In $1960 \mathrm{H}$. Yamabe [28] proposed a program to find optimal metrics in a conformal class on a manifold of dimension at least three by minimizing the total scalar curvature functional, obtaining a constant scalar curvature representative in each conformal class. This program, which now bears his name, led to many advancements by N. Trudinger [26], T. Aubin [3], R. Schoen [25], and many others in the understanding of how geometry, topology and analysis interact with each other in compact Riemannian manifolds. The reader can find an excellent survey of the resolution of the original Yamabe problem in [17. The lack of compactness of the group of conformal transformations of the sphere presents one of the many complications in carrying out Yamabe's program. This same lack of compactness forces one to examine singular solutions which blow up along a closed subset. Many people continue to study both the regular and the singular Yamabe problems, and many open questions in both programs remain. More recent results include [19], in which the authors prove the set of solutions to the Yamabe problem within a conformal class is compact provided $3 \leq n \leq 24$ and the conformal class is not the one of the round sphere.

2000 Mathematics Subject Classification. 35J60, 35B09, 35J30, 35B40.

Key words and phrases. Paneitz operator, Q-curvature, Critical exponent, Isolated singularities, Compactness, Pohozaev invariant.

Research supported in part by Conselho Nacional de Desenvolvimento Científico e Tecnológico (CNPq): grant 305726/2017-0, Coordenação de Aperfeiçoamento de Pessoal de Nível Superior (CAPES): grant 88882.440505/2019-01, and Fundação de Apoio à Pesquisa do Estado de São Paulo (FAPESP): grant 2020/07566-3. 
In recent years many people have pursued parts of Yamabe's program for other notions of curvature. In the present note, we explore a part of the singular Yamabe program as applied to the fourth order $Q$-curvature, which is a higher order analog of scalar curvature. On a Riemannian manifold $(M, g)$ of dimension $n \geq 5$, the $Q$ curvature is

$$
Q_{g}=-\frac{1}{2(n-1)} \Delta_{g} R_{g}-\frac{2}{(n-2)^{2}}\left|\operatorname{Ric}_{g}\right|^{2}+\frac{n^{3}-4 n^{2}+16 n-16}{8(n-1)^{2}(n-2)^{2}} R_{g}^{2},
$$

where $R_{g}$ is the scalar curvature of $g$, $\operatorname{Ric}_{g}$ is the Ricci curvature of $g$, and $\Delta_{g}$ is the Laplace-Beltrami operator of $g$. After a conformal change, the $Q$-curvature transforms as

$$
\widetilde{g}=u^{\frac{4}{n-4}} g \Rightarrow Q_{\widetilde{g}}=\frac{2}{n-4} u^{-\frac{n+4}{n-4}} P_{g} u,
$$

where $P_{g}$ is the Paneitz operator

$$
P_{g} u=\Delta_{g}^{2} u+\operatorname{div}\left(\frac{4}{n-2} \operatorname{Ric}_{g}(\nabla u, \cdot)-\frac{(n-2)^{2}+4}{2(n-1)(n-2)} R_{g}\langle\nabla u, \cdot\rangle\right)+\frac{n-4}{2} Q_{g} u .
$$

Paneitz [22] first discovered the operator $P_{g}$ and investigated its conformal invariance. Thereafter Branson [5, 6] began a thorough investigation of $Q_{g}$ and its variants. The reader can find excellent summaries of the fourth order $Q$-curvature in [7, 9, 14].

The $Q$-curvature of the round metric $\stackrel{\circ}{g}$ is $\frac{n\left(n^{2}-4\right)}{8}$, and setting $Q_{g}$ to be this value gives the equation

$$
P_{g} u=\frac{n(n-4)\left(n^{2}-4\right)}{16} u^{\frac{n+4}{n-4}} .
$$

Just as in the scalar curvature setting, one can search for constant $Q$-curvature metrics in a conformal class by minimizing the total $Q$-curvature. However, because of the conformal invariance one encounters the same lack of compactness and presence of singular solutions. Hang and Yang [15] carry out part of this program in the regular case, assuming that the background metric also has positive Yamabe invariant.

In any event, a complete understanding of the fourth order analog of the Yamabe problem would require an understanding of the following singular problem: let $(M, g)$ be a compact Riemannian manifold and let $\Lambda \subset M$ be a closed subset. A conformal metric $\widetilde{g}=u^{\frac{4}{n-4}} g$ is a singular constant $Q$-curvature metric if $Q_{\widetilde{g}}$ is constant and $\widetilde{g}$ is complete on $M \backslash \Lambda$. According to (2) we can write this geometric problem as

$$
\begin{aligned}
P_{g} u & =\frac{n(n-4)\left(n^{2}-4\right)}{16} u^{\frac{n+4}{n-4}} \text { on } M \backslash \Lambda, \\
\liminf _{x \rightarrow x_{0}} u(x) & =\infty \text { for each } x_{0} \in \Lambda .
\end{aligned}
$$

For the remainder of our work we concentrate on the case that $(M, g)=\left(\mathbf{S}^{n}, \stackrel{\circ}{g}\right)$ is the round metric on the sphere and $\Lambda=\left\{p_{1}, \ldots, p_{k}\right\}$ is a finite set of distinct points. Thus we examine, given a singular set $\Lambda$ with $\#(\Lambda)=k$, the set of functions

$$
u: \mathbf{S}^{n} \backslash \Lambda=\mathbf{S}^{n} \backslash\left\{p_{1}, \ldots, p_{k}\right\} \rightarrow(0, \infty)
$$


that satisfy

$$
\begin{aligned}
\stackrel{\circ}{P} u & =P_{g} u=\frac{n(n-4)\left(n^{2}-4\right)}{16} u^{\frac{n+4}{n-4}} \\
\liminf _{x \rightarrow p_{j}} u(x) & =\infty \text { for each } j=1,2, \ldots, k .
\end{aligned}
$$

For technical reasons we will also require $R_{g} \geq 0$.

Following [21] we define the marked moduli space

$$
\mathcal{M}_{\Lambda}=\left\{g \in[\stackrel{\circ}{g}]: Q_{g}=\frac{n\left(n^{2}-4\right)}{8}, R_{g} \geq 0, g \text { is complete on } \mathbf{S}^{n} \backslash \Lambda\right\}
$$

and the unmarked moduli space

$$
\mathcal{M}_{k}=\left\{g \in[\stackrel{\circ}{g}]: Q_{g}=\frac{n\left(n^{2}-4\right)}{8}, R_{g} \geq 0, g \text { is complete on } \mathbf{S}^{n} \backslash \Lambda, \#(\Lambda)=k\right\} .
$$

We equip each moduli space with the Gromov-Hausdorff topology. C. S. Lin [20] proved that $\mathcal{M}_{1}$ is the empty set, and recently Frank and König [11] classified all metrics in $\mathcal{M}_{2}$, proving

$$
\mathcal{M}_{p, q}=\left(0, \epsilon_{n}\right] \text { for each pair } p \neq q \in \mathbf{S}^{n},
$$

where

$$
\epsilon_{n}=\left(\frac{n(n-4)}{n^{2}-4}\right)^{\frac{n-4}{8}} \in(0,1) .
$$

It follows that

$$
\mathcal{M}_{2}=\left(0, \epsilon_{n}\right] \times\left(\left(\mathbf{S}^{n} \times \mathbf{S}^{n} \backslash \operatorname{diag}\right) / S O(n+1,1)\right),
$$

where the group $S O(n+1,1)$ of conformal transformations acts on each $\mathbf{S}^{n}$ factor simultaneously. These metrics corresponding to a doubly punctured sphere are all rotationally invariant, and are called the Delaunay metrics. We describe them in detail in Section 2.1.

In the present work we explore some of the structure of $\mathcal{M}_{k}$ when $k \geq 3$. Let $\Lambda=\left\{p_{1}, \ldots, p_{k}\right\}$ with $k \geq 3$ and let $g=u^{\frac{4}{n-4}} \stackrel{\circ}{g} \in \mathcal{M}_{\Lambda}$. As it happens, the metric $g$ is asymptotic to a Delaunay metric near each puncture $p_{j}$, and so one can associate a Delaunay parameter $\epsilon_{j}(g) \in\left(0, \epsilon_{n}\right]$ to each $p_{j}$ and $g \in \mathcal{M}_{\Lambda}$. (See Section [2.2.) Our main compactness theorem is the following.

Theorem 1. Let $k \geq 3$ and let $\delta_{1}>0, \delta_{2}>0$ be positive numbers. Then the set

$$
\Omega_{\delta_{1}, \delta_{2}}=\left\{g \in \mathcal{M}_{k}: \operatorname{dist}_{g}\left(p_{j}, p_{l}\right) \geq \delta_{1} \text { for each } j \neq l, \epsilon_{j}(g) \geq \delta_{2}\right\}
$$

is sequentially compact in the Gromov-Hausdorff topology.

We model this result on a compactness theorem of Pollack [23], which states that the similarly defined set in the moduli space of singular Yamabe metrics is sequentially compact. Very recently Wei [27] proved a similar theorem in the context of constant $\sigma_{k^{-}}$ curvature. Pollack's theorem was an important first step in understanding the structure of the moduli space of singular Yamabe metrics on a finitely puctured sphere, a program that is still not complete. We hope our theorem above can play a similar role in advancing the general theory of constant $Q$-curvature metrics with isolated singularities. 


\section{Preliminaries}

In this section we present some prerequisite analysis proven elsewhere which we will use below.

We first rewrite (6) . Pulling back by (the inverse of) stereographic projection, we can write

$$
\stackrel{\circ}{g}=\left(\frac{2}{1+|x|^{2}}\right)^{2} \delta=U_{\mathrm{sph}}^{\frac{4}{n-4}} \delta, \quad U_{\mathrm{sph}}=\left(\frac{1+|x|^{2}}{2}\right)^{\frac{4-n}{2}} .
$$

In these coordinates (6) takes the form

$$
u: \mathbf{R}^{n} \backslash\left\{q_{1}, \ldots, q_{k}\right\} \rightarrow(0, \infty), \quad \Delta_{0}^{2}\left(U_{\mathrm{sph}} u\right)=\frac{n(n-4)\left(n^{2}-4\right)}{16}\left(U_{\mathrm{sph}} u\right)^{\frac{n+4}{n-4}},
$$

where $\Delta_{0}$ is the usual flat Laplacian and $q_{j}$ is the image of $p_{j}$ under the stereographic map. Also, the condition $R_{g} \geq 0$ is equivalent to the differential inequality

$$
-\Delta_{0}\left(U_{\mathrm{sph}} u\right)^{\frac{n-2}{n-4}} \geq 0 \Leftrightarrow-\Delta_{0}\left(U_{\mathrm{sph}} u\right) \geq \frac{2}{n-4} \frac{\left|\nabla\left(U_{\mathrm{sph}} u\right)\right|^{2}}{U_{\mathrm{sph}} u} .
$$

In this Euclidean setting the transformation rule (2) reads

$$
\Delta_{0}^{2} u=A u^{\frac{n+4}{n-4}} \Rightarrow u_{\lambda}(x)=\lambda^{\frac{n-4}{2}} u(\lambda x) \text { satsfies } \Delta_{0}^{2} u_{\lambda}=A u_{\lambda}^{\frac{n+4}{n-4}} \text { for each } \lambda>0
$$

for any constant $A$.

2.1. Delaunay metrics. Let $p \neq q \in \mathbf{S}^{n}$ and let $g \in \mathcal{M}_{\{p, q\}}$. We may precompose by an appropriate dilation and assume $p=-q$, and then rotate $\mathbf{S}^{n}$ so that $p$ is the north pole and $q$ is the south pole. After reframing as in the previous paragraph we obtain a function $u: \mathbf{R}^{n} \backslash\{0\} \rightarrow(0, \infty)$ satisfying the PDE (11). Lin [20] proved that this solution must be rotationally invariant about 0, and later Frank and König [11] classified all the ODE solutions.

Their classification is easiest to see after changing to cylindrical coordinates. We let

$$
\begin{aligned}
t=-\log |x|, & \theta=\frac{x}{|x|}, \\
v(t, \theta) & =e^{\left(\frac{4-n}{2}\right) t} U_{\mathrm{sph}}\left(e^{-t} \theta\right) u\left(e^{-t} \theta\right)=\left(e^{t} \cosh t\right)^{\frac{4-n}{2}} u\left(e^{-t} \theta\right)
\end{aligned}
$$

This transforms the Paneitz operator into

$$
\begin{aligned}
P_{\mathrm{cyl}}= & \frac{\partial^{4}}{\partial t^{4}}+\Delta_{\theta}^{2}+2 \Delta_{\theta} \frac{\partial^{2}}{\partial t^{2}}-\left(\frac{n(n-4)+8}{2}\right) \frac{\partial^{2}}{\partial t^{2}} \\
& -\frac{n(n-4)}{2} \Delta_{\theta}+\frac{n^{2}(n-4)^{2}}{16}
\end{aligned}
$$

so that (11) becomes

$$
v: \mathbf{R} \times \mathbf{S}^{n-1} \rightarrow(0, \infty), \quad P_{\mathrm{cyl}} v=\frac{n(n-4)\left(n^{2}-4\right)}{16} v^{\frac{n+4}{n-4}}
$$


The fact that the orginal function $u$ is radial implies $v$ is a function of $t$ alone, and so (16) reduces to the ODE

$$
\dddot{v}-\left(\frac{n(n-4)+8}{2}\right) \ddot{v}+\frac{n^{2}(n-4)^{2}}{16} v=\frac{n(n-4)\left(n^{2}-4\right)}{16} v^{\frac{n+4}{n-4}} .
$$

We find two solutions explicitly. The cylindrical solution is the only constant solution, namely $v_{\text {cyl }}=\epsilon_{n}$, given in (9). Also, the spherical solution $U_{\text {sph }}$ given in (10) transforms under the change of variables (14) into $v_{\mathrm{sph}}=(\cosh t)^{\frac{4-n}{2}}$. The Delaunay solutions found by Frank and König in [11] interpolate between the cylindrical and spherical solutions. Indeed, for each $\epsilon \in\left(0, \epsilon_{n}\right)$ there exists a unique solution $v_{\epsilon}$ of the ODE (17) realizing its minimal value of $\epsilon$ at $t=0$. Each $v_{\epsilon}$ is periodic with minimal period $T_{\epsilon}$, and these Delaunay solutions account for all global solutions of the ODE (17).

Transforming back to Euclidean coordinates, we of course obtain the solutions

$$
u_{\epsilon}: \mathbf{R}^{n} \backslash\{0\} \rightarrow(0, \infty), \quad u_{\epsilon}(x)=|x|^{\frac{4-n}{2}} v_{\epsilon}(-\log |x|) .
$$

We may then apply global conformal transformations to construct the translated Delaunay solutions. The first such family is $\widetilde{u}_{\epsilon, a}(x)=u_{\epsilon}(x-a)$ for some fixed vector $a \in \mathbf{R}^{n}$. The second family is more important to our later analysis, and is given by translating the point at infinity. More precisely, we define

$$
\begin{aligned}
u_{\epsilon, a}(x) & =\widehat{\mathbb{K}}_{0}\left(\widehat{\mathbb{K}}_{0}\left(u_{\epsilon}(\cdot-a)\right)(x)\right. \\
& =|x|^{\frac{n-4}{2}}\left|\frac{x}{|x|}-\right| x|a|^{\frac{4-n}{2}} v_{\epsilon}\left(-\log |x|-\log \left|\frac{x}{|x|}-\right| x|a|\right),
\end{aligned}
$$

where

$$
\widehat{\mathbb{K}}_{0}(u)(x)=|x|^{4-n} u\left(\frac{x}{|x|^{2}}\right)
$$

is the Kelvin transform of $u$. In cylindrical coordinates we can write this expression for $u_{\epsilon, a}$ as

$$
\begin{aligned}
v_{\epsilon, a}(t, \theta) & =\left|\theta-e^{-t} a\right|^{\frac{4-n}{2}} v_{\epsilon}\left(t+\log \left|\theta-e^{-t} a\right|\right) \\
& =v_{\epsilon}(t)+e^{-t}\langle\theta, a\rangle\left(-\dot{v}_{\epsilon}(t)+\frac{n-4}{2} v_{\epsilon}(t)\right)+\mathcal{O}\left(e^{-2 t}\right)
\end{aligned}
$$

2.2. Asymptotics. In [16] Jin and Xiong proved that any positive, superharmonic solution of (11) in a punctured ball is asymptotically symmetric. In other words, they show there exists $\alpha>0$ such that

$$
u(x)=\bar{u}(|x|)\left(1+\mathcal{O}\left(|x|^{\alpha}\right)\right), \quad \bar{u}(r)=\frac{1}{r^{n-1}\left|\mathbf{S}^{n-1}\right|} \int_{|x|=r} u(\theta) d \theta .
$$

Later the first two authors [2] and the third author [24] independently derived refined asymptotics for positive, singular solutions of (11). Roughly speaking, the translated Delaunay solutions of (19) give the next order term in the expansion of $u$. They show 
there exists $\beta>1, \epsilon \in\left(0, \epsilon_{n}\right], T \in\left[0, T_{\epsilon}\right)$, and $a \in \mathbf{R}^{n}$ such that

$$
\begin{aligned}
u(x) & =|x|^{\frac{4-n}{2}}\left(v_{\epsilon}(-\log |x|+T)\right. \\
& \left.+|x|\left\langle\frac{x}{|x|}, a\right\rangle\left(-\dot{v}_{\epsilon}(-\log |x|+T)+\frac{n-4}{2} v_{\epsilon}(-\log |x|+T)\right)+\mathcal{O}\left(|x|^{\beta}\right)\right) .
\end{aligned}
$$

In cylindrical coordinates this estimate has the form

$$
v(t, \theta)=v_{\epsilon}(t+T)+e^{-t}\langle\theta, a\rangle\left(-\dot{v}_{\epsilon}(t+T)+\frac{n-4}{2} v_{\epsilon}(t+T)\right)+\mathcal{O}\left(e^{-\beta t}\right) .
$$

2.3. Some other useful theorems. For the sake of completeness, we state some background results which will be required later in the proof of our main result.

We first quote the following theorem of Chang, Han and Yang [10, Theorem 1.1].

Theorem 2 (Chang, Han and Yang). Let $n \geq 5$, let $\Lambda \subset \mathbf{S}^{n}$ be a proper closed set, and let $g=u^{\frac{4}{n-4} \stackrel{\circ}{g}}$ be a complete metric on $\mathbf{S}^{n} \backslash \Lambda$ such that

$$
Q_{g}=\frac{n\left(n^{2}-4\right)}{8}, \quad R_{g} \geq 0 .
$$

Then $\partial \stackrel{\circ}{\mathbf{B}}_{\rho}\left(x_{0}\right)$ is has positive mean curvature with respect to $g$, computed with the inward pointing normal, where $\stackrel{\circ}{\mathbf{B}}_{\rho}\left(x_{0}\right)$ is any ball with respect to the round metric contained in $\mathbf{S}^{n} \backslash \Lambda$.

We will also need a version of Harnack's inequality, which was proven by Caristi and Mitidieri [8, Theorem 3.6].

Theorem 3 (Caristi and Mitidieri). Let $u$ be a superharmonic function defined in a domain $\Omega \subset \mathbf{R}^{n}$ such that $\Delta_{0}^{2} u=f(u)$, where $f$ is either linear or superlinear and $f(0)=0$. Then there exists $\rho_{0}>0$ such that for $\rho \leq \rho_{0}$ we have

$$
\begin{aligned}
& \sup u \leq C \inf u \text {, } \\
& \stackrel{\circ}{\mathbf{B}}_{\rho}(p) \quad \stackrel{\circ}{\mathbf{B}}_{\rho}(p)
\end{aligned}
$$

where the constant $c$ depends only on the domain $\Omega$, the function $f$, and $\rho$.

Gursky and Malchiodi [12, Proposition 2.5] prove the existence of a positive Greens function for the Paneitz operator of the round sphere.

Theorem 4 (Gursky and Malchiodi). Let $(M, g)$ be a compact Riemannian manifold such that $R_{g} \geq 0$ and $Q_{g}>0$. Then for each $p \in M$ there exists a Greens function $G_{p}$ satisfying

$$
G_{p}: M \backslash\{p\} \rightarrow(0, \infty), \quad P_{g} G_{p}=\delta_{p},
$$

where $\delta_{p}$ is the Dirac $\delta$-function with a singularity at $p$. Furthermore, if either $n=5,6,7$ or $g$ is conformally flat then there exists $c>0$ depending only on $n$ and $\alpha$ such that

$$
G_{p}(x)=\frac{1}{2 n(n-2)(n-4) \omega_{n}}\left(\operatorname{dist}_{g}(x, p)\right)^{4-n}+\mathcal{O}(1)
$$

in conformal normal coordinates, where $\omega_{n}$ is the volume of a unit ball in $\mathbf{R}^{n}$. 


\section{Pohozaev invariants}

One often finds integral invariants in geometric variational problems. The reader can find a general abstract framework for constructing these invariants in the paper by Gover and Ørsted [13]. In future work we will explicitly write out the full Pohozaev invariant using the first variation tensor defined in [18].

We consider a function

$$
v:(a, b) \times \mathbf{S}^{n-1} \rightarrow \mathbf{R}
$$

satisfying (16). Given such a function $v$ we define the Hamiltonian functional

$$
\begin{aligned}
\mathcal{H}(v)= & -\frac{\partial v}{\partial t} \frac{\partial^{3} v}{\partial t^{3}}+\frac{1}{2}\left(\frac{\partial^{2} v}{\partial t^{2}}\right)^{2}-\frac{1}{2}\left(\Delta_{\theta} v\right)^{2}-\left|\nabla_{\theta} \frac{\partial v}{\partial t}\right|^{2}+\frac{n(n-4)}{4}\left|\nabla_{\theta} v\right|^{2} \\
& +\left(\frac{n(n-4)+8}{4}\right)\left(\frac{\partial v}{\partial t}\right)^{2}-\frac{n^{2}(n-4)^{2}}{32} v^{2}+\frac{(n-4)^{2}\left(n^{2}-4\right)}{32} v^{\frac{2 n}{n-4}} .
\end{aligned}
$$

Integrating by parts we find

$$
\frac{d}{d t} \int_{\{t\} \times \mathbf{S}^{n-1}} \mathcal{H}(v) d \theta=0,
$$

which allows us to define our first integral invariant as

$$
\widetilde{\mathcal{P}}_{\text {rad }}(v)=\int_{\{t\} \times \mathbf{S}^{n-1}} \mathcal{H}(v) d \theta .
$$

Now we can define the radial (or dilational) Pohozaev invariants associated to a metric $g \in \mathcal{M}_{k}$ at each puncture point $p_{j}$. Recall that $g=u^{\frac{4}{n-4}} \stackrel{\circ}{g}$ is a complete, conformally flat metric on $\mathbf{S}^{n} \backslash\left\{p_{1}, \ldots, p_{k}\right\}$ with $Q_{g}=\frac{n\left(n^{2}-4\right)}{8}$ and $R_{g} \geq 0$. Completeness forces

$$
\liminf _{\text {disto }_{g}\left(x, p_{j}\right) \rightarrow 0} u(x)=\infty
$$

for each $j$, while $Q_{g}=\frac{n\left(n^{2}-4\right)}{8}$ is equivalent to the PDE (11), after stereographic projection down to $\mathbf{R}^{n} \backslash\left\{p_{1}, \ldots, p_{k}\right\}$. Choose coordinates centered at one of the punctures $p_{j}$, and the perform the cylindrical change of variables (14), which gives us a function

$$
v:(A, \infty) \times \mathbf{S}^{n-1} \rightarrow(0, \infty)
$$

satisfying (16). We define

$$
\mathcal{P}_{\text {rad }}\left(g, p_{j}\right)=\widetilde{\mathcal{P}}_{\text {rad }}(v)=\int_{\{t\} \times \mathbf{S}^{n-1}} \mathcal{H}(v) d \theta,
$$

which is well-defined by (26) .

In the special case that $k=2$ we can evaluate the dilational Pohozaev invariant more explicitly. In this situation we may as well let the puncture points be the north and south poles, and thus we obtain a function

$$
v=v_{\epsilon}: \mathbf{R} \times \mathbf{S}^{n-1} \rightarrow(0, \infty)
$$


satisfying (17). Thus the Hamiltonian (25) reduces to

$$
\overline{\mathcal{H}}(v)=-\dot{v} \dddot{v}+\frac{1}{2} \ddot{v}^{2}+\left(\frac{n(n-4)+8}{4}\right) \dot{v}^{2}-\frac{n^{2}(n-4)^{2}}{32} v^{2}-\frac{(n-4)^{2}\left(n^{2}-4\right)}{32} v^{\frac{2 n}{n-4}} .
$$

Moreover, because $\overline{\mathcal{H}}$ does not depend on $\theta$ and its integral over a sphere does not depend on $t$, this reduced Hamiltonian must be constant on solutions of (17). (One can, of course, explicitly verify this constancy by taking a derivative.)

A computation reveals

$$
\overline{\mathcal{H}}\left(v_{\mathrm{sph}}\right)=0, \quad \overline{\mathcal{H}}\left(v_{\epsilon_{n}}\right)=-\frac{(n-4)\left(n^{2}-4\right)}{8}\left(\frac{n(n-4)}{n^{2}-4}\right)^{n / 4}<0 .
$$

Furthermore, Proposition 6 of [4] implies the Delaunay solutions are ordered (in fact, uniquely determined!) by their energy (28).

Combining our analysis above with (21) and (22) we immediately see the following

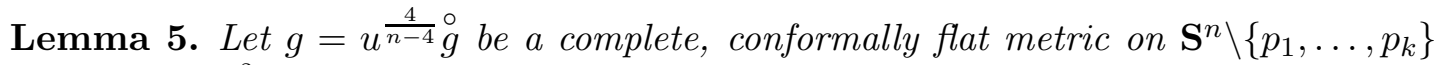
with $Q_{g}=\frac{n\left(n^{2}-4\right)}{8}$ and $R_{g} \geq 0$. For each puncture $p_{j}$ define $\mathcal{P}_{\text {rad }}\left(g, p_{j}\right)$ as above. Then $\mathcal{P}_{\text {rad }}\left(g, p_{j}\right)<0$ and depends only on the necksize $\epsilon_{j}$ of the Delaunay asymptote at $p_{j}$. Moreover, decreasing $\epsilon_{j}$ will increase $\mathcal{P}_{\text {rad }}\left(g, p_{j}\right)$ towards 0 . In particular, bounding the radial Pohozaev invariants $\mathcal{P}_{\text {rad }}\left(g, p_{j}\right)$ away from zero is equivalent to bounding the necksizes $\epsilon_{j}$ away from zero.

Proof. We have shown that

$$
\mathcal{P}_{\text {rad }}\left(g, p_{j}\right)=\int_{\{t\} \times \mathbf{S}^{n-1}} \mathcal{H}(v) d \theta
$$

is well-defined, because the integral does not depend on our choice of $t$. Now let $t \rightarrow \infty$, and observe that $v \rightarrow v_{\epsilon_{j}}$, the Delaunay asymptote of $g$ at the puncture $p_{j}$. In particular, $\mathcal{H}(v) \rightarrow \overline{\mathcal{H}}\left(v_{\epsilon_{j}}\right)$, We conclude that

$$
\mathcal{P}_{\text {rad }}(v)=\mathcal{P}_{\text {rad }}\left(v_{\epsilon_{j}}\right)=\left|\mathbf{S}^{n-1}\right| \overline{\mathcal{H}}\left(v_{\epsilon_{j}}\right)<0 .
$$

The remainder of the lemma follows from the energy ordering theorem of van den Berg [4] applied to the Delaunay solutions, as described in the paragraph above.

Remark 1. Our radial Pohozaev invariant is basically the same as the one defined in Proposition 4.1 of [1]. Jin and Xiong [16] write out the same invariant for higher order equations.

It will actually be useful for later computations to decompose the Hamiltonian energy $\mathcal{H}$ given in (25) as

$$
\mathcal{H}(v)=\mathcal{H}_{\mathrm{cyl}}(v)+\frac{(n-4)^{2}\left(n^{2}-4\right)}{32} v^{\frac{2 n}{n-4}}
$$

The same computation as in (27) shows the following lemma.

Lemma 6. Let $v$ satisfy

$$
v:(a, b) \times \mathbf{S}^{n-1} \rightarrow \mathbf{R}, \quad P_{c y l} v=A v^{\frac{n+4}{n-4}}
$$


for some constant $A$. Then the integral

$$
\int_{\{t\} \times \mathbf{S}^{n-1}} \mathcal{H}_{c y l}(v)+\frac{(n-4)}{2 n} A v^{\frac{2 n}{n-4}} d \theta
$$

does not depend on $t$.

\section{Proof of the compactness theorem}

In this section we prove Theorem 1. We first use standard blow-up techniques to prove a priori bounds on the $\mathcal{C}^{4}$-norm of solutions of (5). Once we obtain these bounds, we use them to extract a convergent subsequence. Finally we prove that our limit is non-trivial, using the fact that the radial Pohozaev invariants of our original sequence of metrics remain bounded away from zero.

4.1. A priori bounds. We prove some a priori bounds for solutions of

$$
\stackrel{\circ}{P} u=\frac{n(n-4)\left(n^{2}-4\right)}{16} u^{\frac{n+4}{n-4}} .
$$

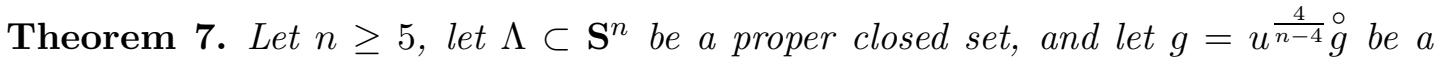
complete metric on $\mathbf{S}^{n} \backslash \Lambda$ such that

$$
Q_{g}=\frac{n\left(n^{2}-4\right)}{8}, \quad R_{g} \geq 0 .
$$

Then there exists $C>0$ depending only on the dimension $n$ such that

$$
u(x) \leq C\left(\operatorname{dist}_{g}(x, \Lambda)\right)^{\frac{4-n}{2}} .
$$

Remark 2. In the context of $g \in \mathcal{M}_{k}$ with $k \geq 2$, our upper bound (30) is very similar to, but slightly stronger than, Proposition 3.2 of [16], because our constant $C$ depends only on the dimension $n$.

Proof. Our proof borrows from Pollack's proof of the corresponding upper bound in the scalar curvature case.

Given any $g$ satisfying the hypotheses of Theorem [7, $x_{0} \notin \Lambda$, and $\rho>0$ such that $\stackrel{\circ}{\mathrm{B}}_{\rho} \subset \mathbf{S}^{n} \backslash \Lambda$ we define the auxiliary function

$$
f: \stackrel{\circ}{\mathbf{B}}_{\rho} \rightarrow \mathbf{R}, \quad f(x)=\left(\rho-\operatorname{dist}_{g}\left(x, x_{0}\right)\right)^{\frac{n-4}{2}} u(x) .
$$

Observe that choosing $\rho=\frac{1}{2} \operatorname{dist}_{g}\left(x_{0}, \Lambda\right)$ yields

$$
f\left(x_{0}\right)=\rho^{\frac{n-4}{2}} u\left(x_{0}\right)=\left(\frac{1}{2} \operatorname{dist}_{g}\left(x_{0}, \Lambda\right)\right)^{\frac{n-4}{2}} u\left(x_{0}\right),
$$

so it will suffice to find $C$ depending only on $n$ such that $f(x) \leq C$ for all admissible choices of $\Lambda, u, x_{0}$, and $\rho$. 
We suppose the contrary and derive a contradiction. To this end, let $\Lambda_{i}, u_{i}, x_{0, i}$ and $\rho_{i}$ be admissible as described above and suppose

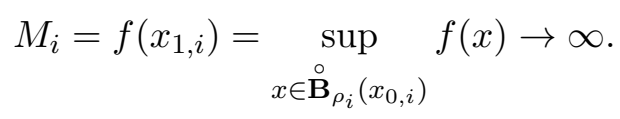

Observe that $\left.f\right|_{\partial{\stackrel{\circ}{\rho_{i}}}_{\rho}\left(x_{0, i}\right)}=0$, so $x_{1, i}$ must lie in the interior of the ball $\stackrel{\circ}{\mathbf{B}}_{\rho_{i}}\left(x_{0, i}\right)$. Next let

$$
r_{i}=\rho_{i}-\operatorname{dist}_{g}\left(x_{1, i}, x_{0, i}\right)
$$

let $y$ be geodesic normal coordinates centered at $x_{1, i}$, and define

$$
\lambda_{i}=2\left(u_{i}\left(x_{1, i}\right)\right)^{\frac{4-n}{2}}, \quad R_{i}=\frac{r_{i}}{\lambda_{i}}=\frac{r_{i}}{2}\left(u_{i}\left(x_{1, i}\right)\right)^{\frac{n-4}{2}}=\frac{1}{2} M_{i}^{\frac{2}{n-4}}
$$

and

$$
w_{i}: \mathbf{B}_{R_{i}}(0) \rightarrow(0, \infty), \quad w_{i}(y)=\lambda_{i}^{\frac{n-4}{2}} u_{i}(\lambda y) .
$$

By (2) (or, equivalently, (13)) the function $w_{i}$ solves

$$
P_{\lambda g} w_{i}=\frac{n(n-4)\left(n^{2}-4\right)}{16} w_{i}^{\frac{n+4}{n-4}}
$$

Moreover, by construction

$$
2^{\frac{n-4}{2}}=w_{i}(0)=\sup _{\mathbf{B}_{R_{i}}(0)} w_{i}(x) .
$$

Using the Arzela-Ascoli theorem we extract a subsequence, which we still denote by $w_{i}$, that converges uniformly on compact subsets of $\mathbf{R}^{n}$. Furthermore, as $i \rightarrow \infty$ the rescaled metrics $\lambda \stackrel{\circ}{g}$ converge to the Euclidean metric. Therefore, in the limit we obtain a function

$$
\bar{w}: \mathbf{R}^{n} \rightarrow[0, \infty), \quad \Delta_{0}^{2} \bar{w}=\frac{n(n-4)\left(n^{2}-4\right)}{16} \bar{w}^{\frac{n+4}{n-4}}, \quad \bar{w}(0)=\sup \bar{w}=2^{\frac{n-4}{2}} .
$$

By the classification theorem in [20, Theorem 1.3] we must have

$$
\bar{w}(x)=\left(\frac{1+|x|^{2}}{2}\right)^{\frac{4-n}{2}} .
$$

Thus each solution $u_{i}$ has a "bubble" when $i$ is sufficiently large, that is for $i$ sufficiently large a small neighborhood of $x_{1, i}$ is close (in $\mathcal{C}^{4}$-norm) to the round metric, and hence has a concave boundary. We verify this by computing the mean curvature of a geodesic sphere explicitly. The round metric has the form $g_{l m}=\frac{4}{\left(1+|x|^{2}\right)^{2}} \delta_{l m}$ in stereographic coordinates, and in general the mean curvature of a hypersurface $\Sigma$ in a Riemannian manifold with unit normal $\eta$ is given by

$$
H_{\Sigma}=-\operatorname{tr}_{g}\left\langle\nabla_{\partial l} \eta, \partial_{m}\right\rangle=-\partial_{l} \eta^{l}-\eta^{p} \Gamma_{l p}^{l} .
$$

A geodesic sphere centered at 0 coincides with a Euclidean round sphere centered at the origin (with a different radius, of course), and so the inward normal vector is

$$
\eta=-\left(\frac{1+|x|^{2}}{2|x|}\right) x^{l} \partial_{l}
$$


A computation shows

$$
H=-2 n|x|\left(1+|x|^{2}\right)+\frac{n-1+n|x|^{2}}{|x|},
$$

which is negative, in particular, when $|x|>3$. Additionally, since $\left\|w_{i}-\bar{w}\right\|_{\mathcal{C}^{4}\left(\mathbf{B}_{\frac{3 R_{i}}{4}}(0)\right)}$ is arbitrarily small when $i$ is sufficiently large, we see that $\partial \mathbf{B}_{\frac{3 R_{i}}{4}}(0)$ is also mean concave

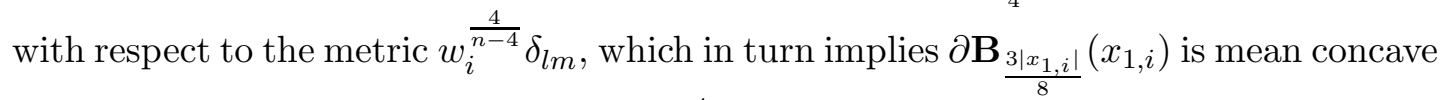
with respect to the metric induced by $u_{i}^{\frac{4}{n-4}} \delta_{l m}$. This contradicts Theorem 2 ,

We immediately obtain the following Corollary.

Corollary 8. For each compact subset $\Omega \subset \mathbf{S}^{n} \backslash \Lambda, l \in \mathbb{N}$ and $\alpha \in(0,1)$ there exists $C_{1}$ depending only on $n, l, \Omega$, and $\alpha$ such that

$$
\left\|u_{i}\right\|_{\mathcal{C}^{l, \alpha}(\Omega)} \leq C_{1}
$$

We also record here a lower bound due to Jin and Xiong [16, Theorem 1.3].

Theorem 9 (Jin and Xiong). Let

$$
v:[A, \infty) \times \mathbf{S}^{n-1} \rightarrow(0, \infty)
$$

solve (16). Then $\mathcal{P}_{\text {rad }}(v) \leq 0$ with equality if and only if

$$
\liminf _{t \rightarrow \infty} v(t, \theta)=\limsup _{t \rightarrow \infty} v(t, \theta)=\lim _{t \rightarrow \infty} v(t, \theta)=0 .
$$

Otherwise, if $\mathcal{P}_{\text {rad }}(v)<0$, there exists $C_{2}>0$ (which depends on the solution v!) such that $v(t, \theta) \geq C_{2}$.

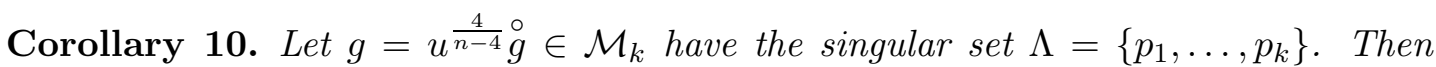
there exists $C_{2}>0$ (depending on the solution u!) such that

$$
u(x) \geq C_{2}\left(\min _{1 \leq j \leq k} \operatorname{dist}_{g}\left(x, p_{j}\right)\right)^{\frac{4-n}{2}} .
$$

4.2. Sequential compactness. In this section we complete our proof of sequential compactness. To this end, let

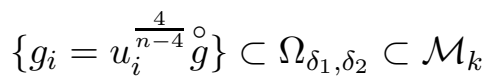

and denote the singular set of the conformal factor $u_{i}$ by $\Lambda_{i}=\left\{p_{1}^{i}, \ldots, p_{k}^{i}\right\}$.

The following lemma will simplify our later analysis since it allows us to assume the singular points are fixed.

Lemma 11. Let $g_{i}=u_{i}^{\frac{4}{n-4}} \stackrel{\circ}{g}$ be a sequence in $\mathcal{M}_{k}$ as described above. After passing to a subsequence, we may assume that when $i$ is sufficiently large both $g_{i}$ and $u_{i}$ are regular on the

$$
\mathbf{S}^{n} \backslash\left(\cup_{j=1}^{k} \stackrel{\circ}{\mathbf{B}}_{\delta_{1} / 2}\left(p_{j}^{i}\right)\right)
$$


where $\stackrel{\circ}{\mathbf{B}}_{r}(p)$ is the geodesic ball centered at $p$ with radius $r$, with respect to the round metric $\stackrel{\circ}{g}$.

Proof. The set

$$
\left(\mathbf{S}^{n}\right)^{k} \backslash\left\{\left(q_{1}, \ldots, q_{k}\right) \in\left(\mathbf{S}^{n}\right)^{k}: \operatorname{dist}_{g}\left(q_{j}, q_{l}\right) \geq \delta_{1} \text { for each } j \neq l\right\}
$$

is compact and contains each singular set $\Lambda_{i}=\left\{p_{1}^{i}, \ldots, p_{k}^{i}\right\}$. Thus we may extract a convergent subsequence, which we still denote as $\Lambda_{i}=\left\{p_{1}^{i}, \ldots, p_{k}^{i}\right\}$, with $p_{j}^{i} \rightarrow \bar{p}_{j}$. The lemma now follows from $p_{j}^{i} \rightarrow \bar{p}_{j}$ for each $j$.

To set notation, we define the compact sets

$$
K_{m}=\mathbf{S}^{n} \backslash\left(\cup_{j=1}^{k}{\stackrel{\circ}{\mathbf{B}_{2}-m} \delta_{1}}_{\left.\left(\bar{p}_{j}\right)\right)}\right.
$$

for each natural number $m \in \mathbf{N}$. By construction the family $\left\{K_{m}\right\}$ is a compact exhaustion of $\mathbf{S}^{n} \backslash\left\{\bar{p}_{1}, \ldots, \bar{p}_{k}\right\}$. Furthermore, by the convergence $p_{j}^{i} \rightarrow \bar{p}_{j}$, for each fixed $m$ there exists $i_{0}$ such that $i \geq i_{0}$ implies $u_{i}$ is smooth in $K_{m}$. Therefore, combining Corollary 8 and the Arzela-Ascoli theorem we obtain a convergent subsequence, which we again denote by $u_{i}$, that converges uniformly on compact subsets of $\mathbf{S}^{n} \backslash \bar{\Lambda}$ to a limit $\bar{u}$, where $\bar{\Lambda}=\left\{\bar{p}_{1}, \ldots, \bar{p}_{k}\right\}$. Furthermore, combining our a priori bounds and elliptic regularity, we see that the limit function satisfies

$$
\bar{u}: \mathbf{S}^{n} \backslash \bar{\Lambda} \rightarrow[0, \infty), \quad \stackrel{\circ}{P} \bar{u}=\frac{n(n-4)\left(n^{2}-4\right)}{16} \bar{u}^{\frac{n+4}{n-4}} .
$$

Proposition 12. The limit function $\bar{u}$ constructed in the paragraph above is positive on $\mathbf{S}^{n} \backslash\left\{\bar{p}_{1}, \ldots, \bar{p}_{k}\right\}$.

Proof. If the proposition does not hold then there exists $q \in \mathbf{S}^{n} \backslash\left\{\bar{p}_{1}, \ldots, \bar{p}_{k}\right\}$ such that

$$
0=\bar{u}(q)=\lim _{i \rightarrow \infty} u_{i}(q)
$$

Let $\epsilon_{i}=u_{i}(q)$ and

$$
w_{i}: \mathbf{S}^{n} \backslash\left\{p_{1}^{i}, \ldots, p_{k}^{i}\right\} \rightarrow(0, \infty), \quad w_{i}(x)=\frac{1}{\epsilon_{i}} u_{i}(x) .
$$

As a consequence of (13), we have

$$
\stackrel{\circ}{P} w_{i}=\epsilon_{i}^{\frac{8}{n-4}} \frac{n(n-4)\left(n^{2}-4\right)}{16} w_{i}^{\frac{n+4}{n-4}} .
$$

In addition, $w_{i}$ satisfies the normalization

$$
w_{i}(q)=1
$$

for each $i$ by construction.

By (38), for each $m \in \mathbf{N}$ there exists $C_{1}$ depending on $m$ and the dimesnion $n$ such that

$$
\sup _{K_{m}} u_{i} \leq C_{1}
$$


Next we find an upper bound for $1 / \epsilon_{i}$. Using the fact that $0<U_{\mathrm{sph}} \leq 2^{\frac{n-4}{2}}$ and the Harnack inequality in Theorem 3 we get

$$
2^{\frac{n-4}{2}} \epsilon_{i} \geq \inf _{K_{m}}\left(U_{\mathrm{sph}} u_{i}\right) \geq \frac{1}{\widetilde{C}(m, n)} \sup _{K_{m}}\left(U_{\mathrm{sph}} u_{i}\right)=C_{2}
$$

and so

$$
\frac{1}{\epsilon_{i}} \leq C_{3}
$$

Combining (43) and (44) we obtain a uniform upper bound

$$
\sup _{K_{m}} w_{i} \leq C_{3},
$$

where $C_{3}$ depends only on $n$ and $m$.

We conclude that $w_{i}$ converges uniformly on compact subsets of $\mathbf{S}^{n} \backslash \bar{\Lambda}$ to a function

$$
\bar{w}: \mathbf{S}^{n} \backslash \bar{\Lambda} \rightarrow[0, \infty), \quad \stackrel{\circ}{P} \bar{w}=0 .
$$

By Theorem 4 we have

$$
\bar{w}=\sum_{j=1}^{k} \alpha_{j} G_{\bar{p}_{j}},
$$

for some coefficients $\alpha_{j} \geq 0$. By the normalization (42) at least one of the $\alpha_{j}$ 's is positive, so (in particular) $\bar{w}$ is a smooth, positive function.

Without loss of generality, we may assume $\alpha_{1} \neq 0$ and center our coordinate system at $\bar{p}_{1}$. We now use the cylindrical coordinates $t=-\log |x|$ and $\theta=\frac{x}{|x|}$ in a punctured ball centered on $\bar{p}_{1}=0$, and define

$$
v_{i}(t, \theta)=e^{\left(\frac{4-n}{2}\right) t} u_{i}\left(e^{-t} \theta\right) U_{\mathrm{sph}}\left(e^{-t} \theta\right), \quad z_{i}(t, \theta)=\frac{1}{\epsilon_{i}} v_{i}(t, \theta)=e^{\left(\frac{4-n}{2}\right) t} w_{i}\left(e^{-t} \theta\right) U_{\mathrm{sph}}\left(e^{-t} \theta\right)
$$

and

$$
\bar{v}(t, \theta)=e^{\left(\frac{4-n}{2}\right) t} \bar{u}\left(e^{-t} \theta\right)(\cosh t)^{\frac{4-n}{2}}, \quad \bar{z}(t, \theta)=e^{\left(\frac{4-n}{2}\right) t} \bar{w}\left(e^{-t} \theta\right)(\cosh t)^{\frac{4-n}{2}} .
$$

By the expansion (24) we have

$$
\begin{aligned}
\bar{z}(t, \theta) & =e^{\left(\frac{4-n}{2}\right) t}(\cosh t)^{\frac{4-n}{2}}\left(\frac{\alpha_{1}}{2 n(n-2)(n-4) \omega_{n}} e^{\left(\frac{n-4}{2}\right) t}+\mathcal{O}(1)\right) \\
& =\frac{\alpha_{1}}{2 n(n-2)(n-4) \omega_{n}}+\mathcal{O}\left(e^{(4-n) t}\right) .
\end{aligned}
$$

Observe that $z_{i}$ satisfies the PDE

$$
P_{\text {cyl }} z_{i}=\epsilon_{i}^{\frac{8}{n-4}} \frac{n(n-4)\left(n^{2}-4\right)}{16} z_{i}^{\frac{n+4}{n-4}}
$$

so, following Lemma 6, the integral

$$
\int_{\{t\} \times \mathbf{S}^{n-1}} \mathcal{H}_{\mathrm{cyl}}\left(z_{i}\right)+\epsilon_{i}^{\frac{8}{n-4}} \frac{(n-4)^{2}\left(n^{2}-4\right)}{32} z_{i}^{\frac{2 n}{n-4}} d \theta
$$


does not depend on $t$. Moreover, taking a limit as $i \rightarrow \infty$, we obtain

$$
\begin{array}{ccc} 
& \lim _{i \rightarrow \infty} \int_{\{t\} \times \mathbf{S}^{n-1}} \mathcal{H}_{\text {cyl }}\left(z_{i}\right)+\epsilon_{i}^{\frac{8}{n-4}} \frac{(n-4)^{2}\left(n^{2}-4\right)}{32} z_{i}^{\frac{2 n}{n-4}} d \theta \\
= & -\int_{\{t\} \times \mathbf{S}^{n-1}} \frac{n^{2}(n-4)^{2}}{32} \cdot \frac{\alpha_{1}^{2}}{4 n^{2}(n-2)^{2}(n-4)^{2} \omega_{n}^{2}}+\mathcal{O}\left(e^{(4-n) t}\right) \\
= & -\frac{n \alpha_{1}^{2}}{128(n-2)^{2} \omega_{n}}+\mathcal{O}\left(e^{(4-n) t}\right) .
\end{array}
$$

On the other hand, by construction

$$
\begin{aligned}
\mathcal{P}_{\text {rad }}\left(v_{i}\right) & =\int_{\{t\} \times \mathbf{S}^{n-1}} \mathcal{H}_{\mathrm{cyl}}\left(v_{i}\right)+\frac{(n-4)^{2}\left(n^{2}-4\right)}{32} v_{i}^{\frac{2 n}{n-4}} d \theta \\
& =\int_{\{t\} \times \mathbf{S}^{n-1}} \epsilon_{i}^{2} \mathcal{H}_{\mathrm{cyl}}\left(z_{i}\right)+\frac{(n-4)^{2}\left(n^{2}-4\right)}{32} \epsilon_{i}^{\frac{2 n}{n-4}} z_{i}^{\frac{2 n}{n-4}} d \theta \rightarrow 0,
\end{aligned}
$$

and so

$$
\lim _{i \rightarrow \infty} \mathcal{P}_{\text {rad }}\left(g_{i}, p_{1}^{i}\right)=0
$$

This contradicts the hypothesis that the asymptotic necksizes of $g_{i}=u_{i}^{\frac{4}{n-4} \stackrel{\circ}{g}}$ at the puncture points $p_{1}^{i}$ are all bounded away from 0 .

We finally complete the proof of Theorem 1 .

Proof. Given a sequence $\left\{g_{i}\right\} \in \Omega_{\delta_{1}, \delta_{2}}$, we have already obtained a limit $\bar{g}=\bar{u}^{\frac{4}{n-4}} \stackrel{\circ}{g}$ as a limit of a subsequence. We know that

$$
\bar{u}: \mathbf{S}^{n} \backslash\left\{\bar{p}_{1}, \ldots, \bar{p}_{k}\right\} \rightarrow(0, \infty), \quad \stackrel{\circ}{P} \bar{u}=\frac{n(n-4)\left(n^{2}-4\right)}{16} \bar{u}^{\frac{n+4}{n-4}},
$$

where $\bar{p}_{j}=\lim _{i \rightarrow \infty} p_{j}^{i}$. We have also shown that $\bar{u}>0$ on $\mathbf{S}^{n} \backslash\left\{\bar{p}_{1}, \ldots, \bar{p}_{k}\right\}$.

It only remains to verify that $\bar{g}$ is complete. If $\bar{g}$ is incomplete then there exists $j \in\{1, \ldots, k\}$ such that

$$
\liminf _{x \rightarrow \bar{p}_{j}} \bar{u}(x)<\infty .
$$

In this case Theorem 9 implies $\mathcal{P}_{\text {rad }}\left(\bar{g}, \bar{p}_{j}\right)=0$. However, by construction

$$
\mathcal{P}_{\text {rad }}\left(\bar{g}, \bar{p}_{j}\right)=\lim _{i \rightarrow \infty} \mathcal{P}_{\text {rad }}\left(g_{i}, p_{j}^{i}\right) \geq \delta_{2}
$$

giving a contradiction. We conclude that $\bar{g}$ is indeed in $\Omega_{\delta_{1}, \delta_{2}}$.

\section{REFERENCES}

[1] M. O. Ahmedou, Z. Djadli, and A. Malchiodi. Prescribing a fourth-order conformal invariant on the standard sphere II: blow-up analysis and applications. Ann. Scuola Norm. Sup. Pisa 5 (2002), $387-434$.

[2] J. H. Andrade and J. M. do Ó. Asymptotics for singular solutions of conformally invariant fourth order systems in the punctured ball. preprint, arXiv:2003.03487.

[3] T. Aubin. Équations différentielles non linéaires et problème de Yamabe concernant la courbure scalaire. J. Math. Pures Appl. 55 (1976), 269-296.

[4] J. van den Berg. The phase-plane picture for a class of fourth-order conservative differential equations. J. Differential Equations. 161 (2000), 110-153. 
[5] T. Branson. Differential operators canonically associated to a conformal structure. Math. Scandinavia. 57 (1985), 293-345.

[6] T. Branson. Group representations arising from Lorentz conformal geometry. J. Funct. Anal. 74 (1987), 199-291.

[7] T. Branson and A. R. Gover. Origins, applications and generalisations of the Q-curvature. Acta Appl. Math. 102 (2008), 131-146.

[8] G. Caristi and E. Mitidieri. Harnack inequalities and applications to solutions of biharmonic equations. Operator Theory: Advances and Applications. 168 (2006), 1-26.

[9] S.-Y. A. Chang, M. Eastwood, B. Ørsted, and P. Yang. What is Q-curvature? Acta Appl. Math. 102 (2008), 119-125.

[10] S.-Y. A. Chang, Z.-C. Han, and P. Yang. Some remarks on the geometry of a class of locally conformally flat metrics. Progress in Math. 333 (2020), 37-56.

[11] R. Frank and T. König. Classification of positive solutions to a nonlinear biharmonic equation with critical exponent. Anal. PDE 12 (2019), 1101-1113.

[12] M. Gursky and A. Malchiodi. A strong maximum principle for the Paneitz operator and a nonlocal flow for the Q-curvature. J. Eur. Math. Soc. 17 (2015), 2137-2173.

[13] A. R. Gover and B. Ørsted. Universal principles for Kazdan-Warner and Pohozaev-Schoen type identities. Comm. Contemp. Math. 15 (2013),

[14] F. Hang and P. Yang. Lectures on the fourth order Q-curvature equation. Geometric analysis around scalar curvature, Lect. Notes Ser. Inst. Math. Sci. Natl. Univ. Singap. 31 (2016), 1-33.

[15] F. Hang and P. Yang. Q-curvature on a class of manifolds with dimension at least 5. Comm. Pure Appl. Math. 69 (2016), 1452-1491.

[16] T. Jin and J. Xiong. Asymptotic symmetry and local behavior of solutions of higher order conformally invariant equations with isolated singularities. preprint, arXiv:1901.01678.

[17] J. Lee and T. Parker. The Yamabe problem. Bull. Amer. Math. Soc. 17 (1987), 37-91.

[18] Y.-J. Lin and W. Yuan. A symmetric 2-tensor cannonically associated to Q-curvature and its applications. Pac. J. Math. 291 (2017) 425-438.

[19] M. Khuri, F. C. Marques, and R. Schoen. A compactness theorem for the Yamabe problem. J. Differential Geom. 81 (2009), 143-196.

[20] C. S. Lin. A classification of solutions of a conformally invariant fourth order equation in $\mathbf{R}^{n}$. Comment. Math. Helv. 73 (1998), 206-231.

[21] R. Mazzeo, D. Pollack, and K. Uhlenbeck. Moduli spaces of singular Yamabe metrics. J. Amer. Math. Soc. 9 (1996), 303-344.

[22] S. Paneitz. A quartic conformally covariant differential operator for arbitrary pseudo-Riemannian manifolds. SIGMA Symmetry Integrability Geom. Methods Appl. 4 (2008), 3 pages (preprint from 1983).

[23] D. Pollack. Compactness results for complete metrics of constant positive scalar curvature on subdomains of $\mathbf{S}^{n}$. Indiana Univ. Math. J. 42 (1993), 1441-1456.

[24] J. Ratzkin. On constant Q-curvature metrics with isolated singularities. preprint, arXiv:2001.07984.

[25] R. Schoen. Conformal deformation of a Riemannian metric to constant scalar curvature. J. Diff. Geom. 20 (1984), 479-495.

[26] N. Trudinger. Remarks concerning the conformal deformation of Riemannian structures on compact manifolds. Ann. Scuola Norm. Pisa. 22 (1968), 265-274.

[27] W. Wei. Compactness theorem of complete $k$-curvature manifolds with isolated singularities. preprint, arxiv.2008.08777.

[28] H. Yamabe. On the deformation of Riemannian structures on a compact manifold. Osaka Math. J. 12 (1960), 21-37. 
(J.H. Andrade) Institute of Mathematics and Statistics, University of São Paulo 05508-090, SÃo PAUlo-SP, Brazil

AND

Department of Mathematics, Federal University of Paraíba 58051-900, JoÃo Pessoa-PB, Brazil

Email address: andradejh@ime.usp.br

Email address: andradejh@mat.ufpb.br

(J.M. do Ó) Department of Mathematics, Federal University of Paraíba 58051-900, JoÃo Pessoa-PB, BrazIL

Email address: jmbo@pq.cnpq.br

(J. Ratzkin) Department of Mathematics, Universität WürzburG 97070, WürzBurG-BA, GERMAnY

Email address: jesse.ratzkin@mathematik.uni-wuerzburg.de 